\title{
Transmission Properties of Phononical Dodecagonal Filter
}

\author{
W. SOCHACKI
}

\author{
Department of Mechanics and Fundamentals of Machinery Design, \\ Czestochowa University of Technology, Poland
}

Doi: 10.12693/APhysPolA.138.328

*e-mail: w.sochacki@imipkm.pcz.pl

\begin{abstract}
The study analyzed mechanical wave propagation in the aperiodic structure of the dodecagonal superlattice made of amorphous $\mathrm{Zr}_{55} \mathrm{Cu}_{30} \mathrm{Ni}_{5} \mathrm{Al}_{10}$ alloy. Methanol, n-Propanol and distilled water with additions of n-Propanol and ethanol were adopted as the second material forming the structure, after which their influence on the structure of wave transmission in the frequency range up to $40 \mathrm{kHz}$ was determined. The analyzed multilayer was surrounded by glass. The change of liquid materials affected the transmission peak shifts. The Transfer Matrix Method (TMM) algorithm was used to investigate the effect of the material on the transmission structure, while using the Finite Difference Time Domain (FDTD) and Discrete Fourier Transform (DFT) algorithms, the power spectra and wave stabilization time after passing through the structure were determined. The study showed the existence of broad phononic bandgap for a dodecagonal structure with a small number of low transmission peaks.
\end{abstract}

topics: finite difference time domain, aperiodic structure, multilayers, phononic, filters

\section{Introduction}

Quasi one-dimensional multi-layer structures, due to their special transmission properties, are used as filters for mechanical wave $[1,2]$ and electromagnetic wave [3]. Depending on the properties of the materials, their distribution in the structure and the thickness of the layers used, for some ranges of the wave frequency, the phononic bandgap (PhBG) may occur in these composites. Numerical analysis of the designed structures allows for the initial prediction of their transmission properties, which allows for a significant reduction of research costs on real systems. In order to determine the properties of phononic systems, many algorithms have been developed to allow their analysis. The analysis of translationally invariant structures is possible using the Bloch theorem. Unfortunately, its use for quasi-periodic or aperiodic structures is not possible. Lack of long-range ordering forces the use of other types of tools, which are characterized by considerable time-consuming. The most commonly used algorithms include Finite Difference Time Domain (FDTD) and Transfer Matrix Method (TMM) algorithm, which were used in this work [4].

Modern technologies allow the production of metallic glasses with special properties. The magnetic properties [5-7], as well as the magnetocaloric effect [8] are very intensely studied. Due to the disordered internal structure, their phononical properties may be interesting. The paper proposes the use of an amorphous alloy $\mathrm{Zr}_{55} \mathrm{Cu}_{30} \mathrm{Ni}_{5} \mathrm{Al}_{10}$ to construct an aperiodic multilayer structure. The transmission properties were analyzed depending on the type of the second material. The structure made

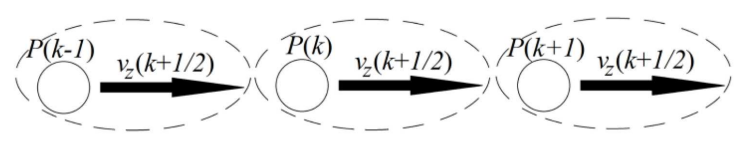

Fig. 1. One-dimensional acoustic unit cell.

of amorphous material was immersed successively in methanol, n-Propanol and in distilled water with additions of n-Propanol or ethanol and surrounded by glass.

\section{Mathematical models}

Pressure waves at time $t$ propagating in the scalar pressure matrix $P$ in space $\boldsymbol{x}$ dependent on vector velocity field $\boldsymbol{v}$ for material with density $\rho$ and speed of mechanical wave propagation $c$ are described by the system of equations:

$$
\left\{\begin{array}{l}
\frac{\partial}{\partial t} P(\boldsymbol{x}, t)=\rho c^{2} \boldsymbol{\nabla} \cdot \boldsymbol{v} \\
\rho \frac{\partial}{\partial t} \boldsymbol{v}(\boldsymbol{x}, t)=\nabla P(\boldsymbol{x}, t)
\end{array}\right.
$$

The mutual position of pressure and the velocity fields in the structure of the one-dimensional FDTD algorithm $[3,8]$ for the discretization index of space $k$, is shown in Fig. 1.

In the FDTD algorithm for a one-dimensional structure in the direction of the axis $z$ and the place at the time specified by the index $n$, the system of equations (1) should be written as

$$
\begin{aligned}
& P^{n+\frac{1}{2}}(k)=P^{n-\frac{1}{2}}(k) \\
& +\frac{\Delta t \rho(k)(c(k))^{2}}{\Delta z}\left[v_{z}^{n}\left(k+\frac{1}{2}\right)-v_{z}^{n}\left(k-\frac{1}{2}\right)\right]
\end{aligned}
$$


and

$$
\begin{aligned}
& v_{z}^{n+1}\left(k+\frac{1}{2}\right)=v_{z}^{n}\left(k+\frac{1}{2}\right) \\
& +\frac{\Delta t}{\Delta z \rho\left(k+\frac{1}{2}\right)}\left[P^{n+\frac{1}{2}}\left(k+\frac{1}{2}\right)-P^{n+\frac{1}{2}}(k)\right]
\end{aligned}
$$

where the time step $\Delta t$ depends on the space step $\Delta z$, and the maximum propagated wave speed $v_{\max }$ in the simulation according to Courant's stability condition

$$
\Delta t \leq \frac{\Delta z}{v_{\max }}
$$

The power spectrum of wave for the input frequency was obtained using the normalized square of the absolute complex values of Discrete Fourier Transform (DFT).

The second method used in the article is the Transfer Matrix Method algorithm [4]. It allows to determine the transmission based on the characteristic matrix of defined n-layered structure, which can be described by

$$
\begin{aligned}
T & =\mid\left[\Phi_{\text {in }, 1}\left[\prod_{i=2}^{n} \Phi_{i-1, i}\left[\begin{array}{cc}
\mathrm{e}^{\frac{\mathrm{i} 2 \pi f d_{i}}{c_{i}}} & 0 \\
0 & \mathrm{e}^{\frac{-\mathrm{i} 2 \pi f c_{i}}{d_{i}}}
\end{array}\right]\right]\right. \\
& \left.\times \Phi_{n, \text { out }}\right]\left._{11}^{-2}\right|^{-2}
\end{aligned}
$$

where $f$ is the frequency, $d_{i}$ is thickness of layer $i$, and transition matrix $\Phi$ between layers is defined by

$$
\Phi_{i, i+1}=\frac{1}{2}\left[\begin{array}{cc}
1+\frac{c_{i+1} \rho_{i+1}}{c_{i} \rho_{i}} & \frac{c_{i+1} \rho_{i+1}}{c_{i} \rho_{i}}-1 \\
1-\frac{c_{i+1} \rho_{i+1}}{c_{i} \rho_{i}} & 1+\frac{c_{i+1} \rho_{i+1}}{c_{i} \rho_{i}}
\end{array}\right] .
$$

\section{Research}

Concatenation rules [10] for a quasi onedimensional dodecagonal structure $\mathrm{X}_{L}^{D}$, for evenand odd-numbered layer $n_{E}$ and $n_{O}$, respectively, are given by:

$$
X_{n_{E}}^{D}=X_{n-1}^{D} X_{n-1}^{D} X_{n-2}^{D},
$$

and

$$
X_{n_{O}}^{D}=X_{n-2}^{D} X_{n-2}^{D} X_{n-2}^{D} X_{n-3}^{D},
$$

with the initial conditions:

$$
X_{0}^{D}=A, \quad X_{1}^{D}=B .
$$

The distribution of layers in a quasi one-dimensional dodecagonal structures for generation number $L$ from 2 to 5 are presented in Table I.

TABLE I

Configuration of the layers of dodecagonal structures.

\begin{tabular}{c|c}
\hline \hline$L$ & $X_{L}^{D}$ \\
\hline 2 & $\mathrm{BBA}$ \\
3 & $\mathrm{BBBA}$ \\
4 & $\mathrm{BBBABBBABBA}$ \\
5 & BBBABBBABBBABBA
\end{tabular}

TABLE II

Material parameters of the components of the multilayer structure [11-15].

\begin{tabular}{l|c|c}
\hline \hline \multicolumn{1}{c|}{ Material } & $\begin{array}{c}\text { Mass density } \\
\rho\left[\mathrm{kg} / \mathrm{m}^{3}\right]\end{array}$ & $\begin{array}{c}\text { Velocity of sound } \\
v[\mathrm{~m} / \mathrm{s}]\end{array}$ \\
\hline $\mathrm{Zr}_{55} \mathrm{Cu}_{30} \mathrm{Ni}_{5} \mathrm{Al}_{10}$ & 6829 & 1633 \\
Distilled water & 998 & 1482 \\
Glass & 3880 & 4000 \\
Metanol & 792 & 1100 \\
n-Propanol & 786 & 1170 \\
$5 \%$ n-Propanol & 992 & 1520 \\
$5 \%$ Etanol & 996 & 1514
\end{tabular}
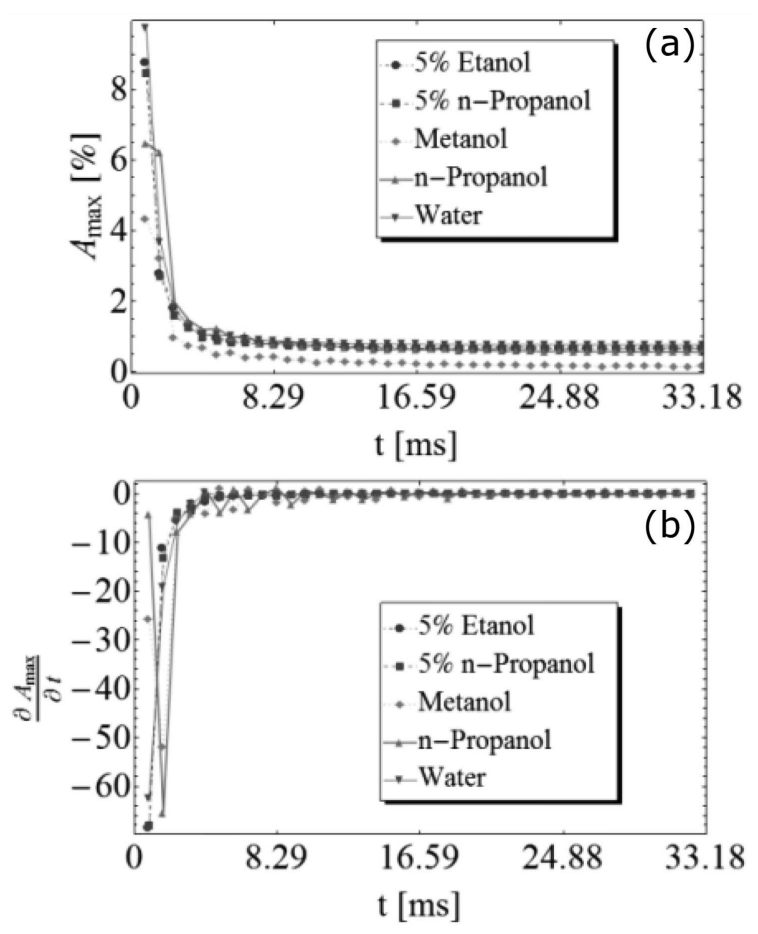

Fig. 2. The value of the maximum amplitude at the time (a) of the samples tested and its derivative (b).

The CBBBABBBABBAC structure for $L=4$ was used in the calculations. As C, the glass layer was determined, $\mathrm{B}$ was the amorphous alloy $\mathrm{Zr}_{55} \mathrm{Cu}_{30} \mathrm{Ni}_{5} \mathrm{Al}_{10}$, whereas $\mathrm{A}$ was a liquid layer which in the calculation was methanol, n-Propanol and distilled water with additions of $5 \%$ n-Propanol or $5 \%$ ethanol.

The material parameters used in the work were collected in Table II. Layer thicknesses dA, dB, and $\mathrm{dC}$ for FDTD and TMM simulations were $33.18 \mathrm{~mm}, 36.29 \mathrm{~mm}$, and $88.89 \mathrm{~mm}$, respectively. The space step $\Delta x$ was $1.66 \mathrm{~mm}$, while at $\Delta t$ from Courant condition was 331.8 ns To determine the stabilization time of the results in the FDTD algorithm, a soft source with the frequency $7751 \mathrm{~Hz}$ was used. The simulation was carried out for $10^{5}$ time steps, which gave the simulation time $33.18 \mathrm{~ms}$. 
The maximum amplitude of the obtained signals for the various liquid materials of analyzed structure is shown in Fig. 2a. And their normalized percentage value of the derivative is shown in Fig. $2 \mathrm{~b}$. The amplitude of the input signal structure has been normalized to $100 \%$. The output amplitudes are the percentage of the input signal. The study determined the average time to stabilize the signal in the simulation and it was $4.98 \mathrm{~ms}$ after 15000 time steps where the derivative value for measurements of the sample with the water layer, as the reference material against which the layer thicknesses were determined, was below $2 \%$.

The use of the TMM algorithm allowed to determine the transmission spectrum of the analyzed structures (Fig. 3a). In all the analyzed structures, the existence of phononic bandgaps in acoustic frequency range of mechanical waves was demonstrated. The low intensity transmission peaks were inside of the bandgaps. Figure $3 \mathrm{~b}$ shows the shift of the transmission peak depending on the liquid used. The transmission for five percent solutions of n-Propanol and ethanol in distilled water for the analyzed frequency range did not differ significantly from the transmission for distilled water. Obtained peaks with a large half-width would not allow unambiguous identification of the admixture. However, differences in the spectra of methanol, n-Propanol and distilled water allow for unambiguous identification of the material used. Applying a superlattice with a higher generation number, and hence increasing the number of layers of the analyzed structure, one could reduce the half-width of transmission peaks and unambiguous identification of the analyzed substance.
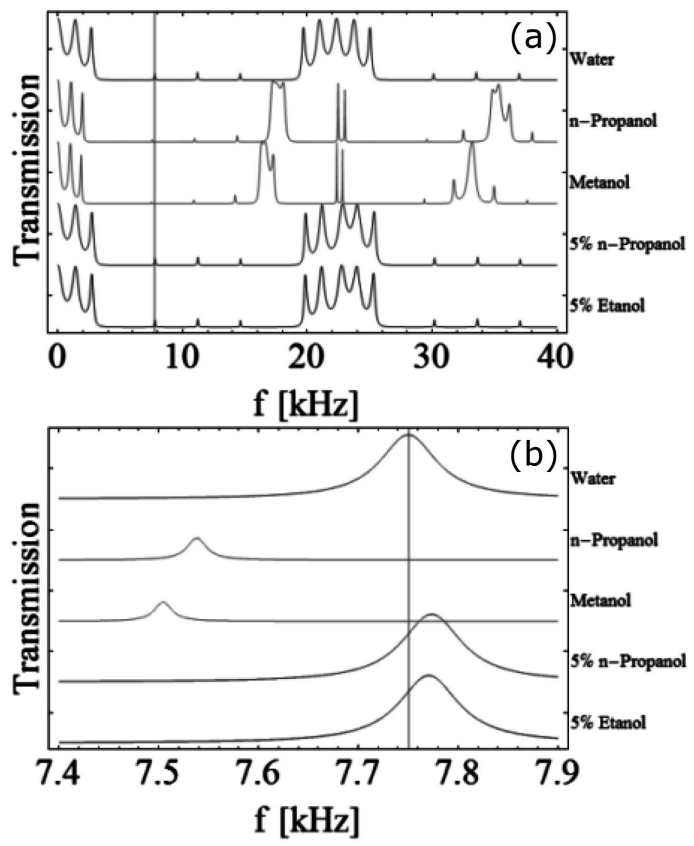

Fig. 3. Transmission spectra of analyzed structures: (a) first low frequency peak inside the bandgap, (b) the grid line is set for $7751 \mathrm{~Hz}$.

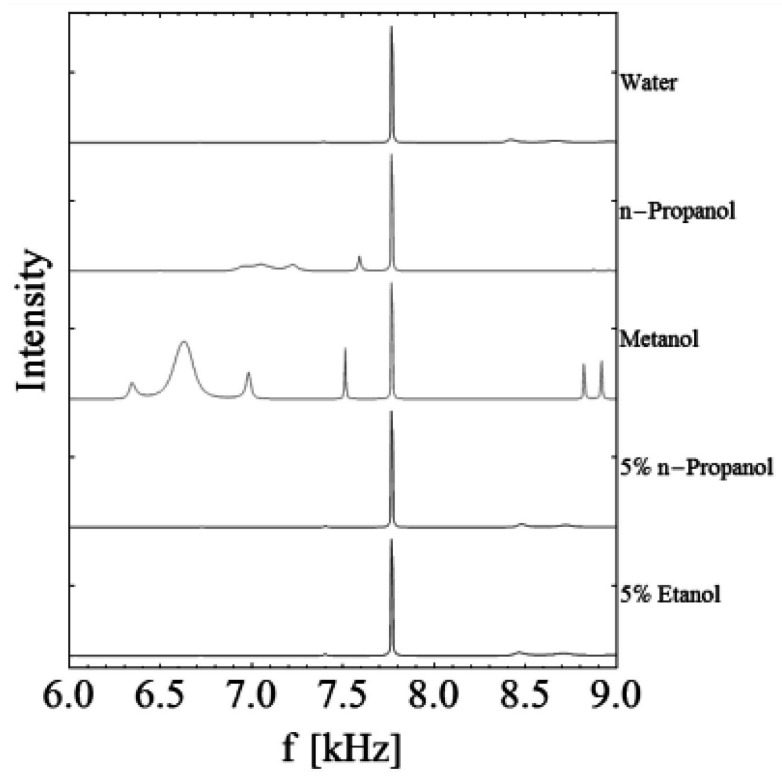

Fig. 4. Power spectrum of analyzed structures.

The frequency of the wave source in the vicinity of the transmission peak, even at low intensity, results in a peak in the power spectrum of the output signal (Fig. 4). In n-Propanol and methanol there was a bandgap for the given frequency, so the output signal was characterized by a much larger frequency range in the power spectrum.

\section{Conclusions}

The study investigated the properties of a filter for mechanical waves built of multi-planar aperiodic dodecagonal structure. The existence of bandgaps for the analyzed structures in a wide range of acoustic frequencies has been demonstrated. It was shown that the material used to build the structure has a significant impact on the transmission of the system. It has been shown that the response of the system for the inertial frequency consists of a larger number of component frequencies.

\section{References}

[1] I.Kriegel, F. Scotognella, Physica E 85, 34 (2017).

[2] S. Villa-Arango, R. Torres, P.A. Kyriacou, R. Lucklum, Measurement 102, 20 (2017).

[3] S.Garus, J.Garus, M.Szota, M.Nabiałek, K.Gruszka, K.Błoch, Arch. Mater. Sci. Eng. 64, 110 (2013).

[4] S. Garus, W. Sochacki, J. Appl. Math. Comput. Mech. 16, 17 (2017).

[5] K. Błoch, M. Nabiałek, M. Dośpiał, S. Garus, Arch. Metall. Mater. 60, 7 (2015). 
[6] K. Gruszka, M. Nabiałek, M. Szota, K. Bloch, J. Gondro, P. Pietrusiewicz, A.V. Sandu, A.M. Mustafa Al Bakri, S. Walters, K. Walters, S. Garus, M. Dośpiał, J. Mizera, Arch. Metall. Mater. 61, 641 (2016).

[7] S. Garus, M. Nabiałek, J. Garus, Acta Phys. Pol. A 126, 960 (2014).

[8] P. Gębara, P. Pawlik, B. Michalski, J.J. Wysłocki, Acta Phys. Pol. A 127, 576 (2015).

[9] D.M. Sullivan, Electromagnetic Simulation Using the FDTD Method, IEEE Press, New York 2000.

[10] J.E.S. Socolar, Phys. Rev. B 39, 10519 (1989).

[11] N. Bilaniuk, G.S.K. Wong, J. Acoust. Soc. Am. 93, 1609 (1993).
[12] N. Bilaniuk, G.S.K. Wong, J. Acoust. Soc. Am. 99, 3257 (1996).

[13] M. Fukuhara X. Wang, A. Inoue, J. NonCryst. Solids 356, 1707 (2010).

[14] S. Villa-Arango, R. Torres Villa, P.A. Kyriacou, R. Lucklum, IEEE Sens. J. 16, 6727 (2016).

[15] S. Villa-Arango, R. Torres, P.A. Kyriacou, R.Lucklum in: VII Latin American Congress on Biomedical Engineering CLAIB 2016, Bucaramanga (Colombia) 2016 IFMBE Proceedings Vol. 60, Eds. I. Torres, J. Bustamante, D. Sierra, Springer, Singapore 2017. 